Meta

Journal des traducteurs

Translators' Journal

\title{
Traduire l'étranger
}

\section{Albert Bensoussan}

Volume 35, numéro 3, septembre 1990

La traduction dans le monde hispanolusophone

URI : https://id.erudit.org/iderudit/002060ar

DOI : https://doi.org/10.7202/002060ar

Aller au sommaire du numéro

Éditeur(s)

Les Presses de l'Université de Montréal

ISSN

0026-0452 (imprimé)

1492-1421 (numérique)

Découvrir la revue

Citer cet article

Bensoussan, A. (1990). Traduire l'étranger. Meta, 35(3), 597-601.

https://doi.org/10.7202/002060ar

Ce document est protégé par la loi sur le droit d'auteur. L'utilisation des services d'Érudit (y compris la reproduction) est assujettie à sa politique d'utilisation que vous pouvez consulter en ligne.

https://apropos.erudit.org/fr/usagers/politique-dutilisation/
Cet article est diffusé et préservé par Érudit.

Érudit est un consortium interuniversitaire sans but lucratif composé de l'Université de Montréal, l'Université Laval et l'Université du Québec à Montréal. Il a pour mission la promotion et la valorisation de la recherche. https://www.erudit.org/fr/ 


\section{TRADUIRE L'ÉTRANGER}

ALBERT BENSOUSSAN

Université de Rennes, France

Ce que nous appelons traduction, ce concept neuf qui consista à faire parler l'Étranger dans une autre langue, est né à la Renaissance. Le mot même de traduction date du XVI e siècle et semble avoir été introduit en français par l'humaniste Étienne Dolet. Nous le trouvons alors couramment sous la plume de Montaigne, de Du Bellay ou d'Amyot, l'illustre traducteur de Plutarque. Le phénix fut le symbole favori de la Renaissance, l'oiseau de feu consumé, cendres fumantes, battant des ailes derechef et reprenant l'envol. Mais fallait-il qu'il mourût pour renaître? La traduction représenta alors un magnifique essor des Anciens retrouvés, transportés dans toutes les langues de l'Europe, imités, plagiés (mais nous n'oublions pas qu'il y eut auparavant de vaillantes entreprises de même encre, de la Bible de Saint-Jérôme aux travaux lumineux de l'École de Tolède). Alors le poète bien né prenait son luth et nous donnait sa version du Collige rosas, du Beatus ille ou du Carpe diem. Et les sonnets de Ronsard supplantaient les odes horaciennes qui avaient elles-mêmes supplanté les odes pindariennes, les églogues de Garcilaso de la Vega prenaient la place des bucoliques virginiennes, Luis de León et Jean de la Croix «actualisaient» les épithalames hébraïques.

Cette imitation ne s'arrêtait pas à la barrière des Antiques. Une véritable boulimie d'écriture s'emparait des copistes, les Modernes s'épiaient, se pillaient. Non content de «transporter» en français Guzmán de Alfarache du grand Mateo Alemán, l'inventeur du roman picaresque espagnol, Le Sage qui, sans faire de manières, ne voulut retenir que la somme des aventures en gommant digressions et discours moraux - le substrat idéologique, en fait - écrivit pour son propre compte d'auteur Gil Blas de Santillane et ce faisant se tailla une part méritée de succès. Moll Flanders devait tout à La pícara Justina, Le Cid de Corneille se parait des plumes de Guillén de Castro, et son Menteur de celles d'Alarcón. Rousseau puisait chez Cervantes, et son discours de l'âge d'or, sa dialectique du «mien» et du «tien» qui allait fonder sa théorie sur l'inégalité. Il est vrai que Cervantes se faisait voler la seconde partie du Don Quichotte par le vulgaire Avellaneda et que la propriété littéraire devait attendre le XIX ${ }^{\mathrm{e}}$ siècle pour s'organiser et se fonder en droit.

Ainsi donc, des siècles durant, s'appropria-t-on l'Étranger. Il allait de soi que Shéhérazade parlât le français châtié de Galland, quoique Mardrus, peu après, lui rendît justice avec une pointe de lubricité et quelques gros mots empruntés à l'arabe. Chez nous, donc, le traducteur, trois siècles durant, s'attacha à franciser, à annexer, à phagocyter, à coloniser l'Étranger.

On appelait Cervantes, Michel et l'Italien Alciati - l'inventeur de l'emblémisme André Alciat. On ne savait plus d'où venait le texte et qui était l'auteur. La culture était à qui voulait ou pouvait la prendre.

Un mauvais traducteur du début du siècle - et c'est pour lui que les Italiens s'écrièrent en un mauvais jeu de mots: traduttore traditore - eut l'idée de traduire en espagnol une comédie boulevardière de Georges de Porto-Riche; l'auteur fut par lui adopté : il le nomma Jorge de Puerto-Rico. On rit, n'est-ce pas? Mais c'est que de nos jours pareille pratique est insoutenable. Le $\mathrm{XX}^{\mathrm{e}}$ siècle a, par deux fois, assis la Société des Nations autour d'une même table, et derrière chaque plénipotentiaire se tenait, 
comme son ombre, un interprète, un truchement, car il fallait que chacun pût parler et se faire entendre à égalité. Cette pratique a profondément modifié l'éthique de la traduction, qu'elle soit littéraire ou pas. Une exigence absolue de fidélité - quoiqu'il faille s'entendre sur la portée exacte de ce terme - s'est assortie d'un respect non moins exigeant de l'Étranger, ou disons de l'étrangeté. Certes la traduction littéraire, au nom même de la fidélité, doit fuir comme la peste le littéral et tendre au littéraire, puisque comme le dit si bien Octavio Paz: «la traduction est une fonction spécialisée de la littérature» (Traducción: literatura y literalidad, Barcelone, Tusquets, 1971).

Il n'est donc pas question de coller, comme par quelque calque, au texte original au point d'hispaniser son texte français: une bévue récente n'a-t-elle pas conduit tel traducteur, par ailleurs excellent, à rendre le dependiente d'une boutique par un curieux dépendant français? En revanche, en l'absence d'équivalent français l'emprunt est toujours possible et l'on peut légitimement parler de «cacique», de «péon» - avec un accent français - tout comme le goût de la couleur locale imposa naguère à Mérimée le «toréador» et la «manzanille».

Ceci dit, s'il est vrai que la traduction est une branche de la littérature, plus qu'un rameau de la linguistique, le traducteur n'a pas pour vocation - comme ce fut le cas à la Renaissance - de se substituer à l'auteur pour faire entendre sa propre voix. Il doit parler comme son auteur, il doit mettre ses pas dans ses pas, enfiler sa veste, chausser ses pantoufles, épier ses tics, guetter ses gestes et restituer, à la façon d'une doublure de théâtre, sa silhouette et les inflexions de sa voix. La traduction n'est-elle pas, comme dit l'écrivain George Borrow, «un écho»? Quoique l'image soit peu gratifiante pour le traducteur. Disons, plus simplement, que le traducteur doit se mettre en condition, doit ambientarse, et je me rappelle à ce propos avoir traduit l'écrivain argentin Néstor Sánchez - dont l'œuvre difficile ne semble être en apparence qu'une savante jonglerie verbale, alors qu'elle est imprégnée d'un argentinisme nourri de nostalgie et d'exil - en me gorgeant de maté.

La plume dans une main, la bombilla - on peut traduire par pipette - de l'autre, ou encore empoignant la chaude calebasse - el mate proprement dit - - je psalmodiais les paroles de Néstor, en quête d'un rythme lointain, d'une musique étrange d'un pays que je ne connaissais pas et qui me parvenait au travers des phrases sinueuses, étirées, incommodes, et qui m'imposaient une réalité comme à côté du réel, en déphasage, un peu comme celles que je découvrirais ensuite en traduisant Juan Carlos Onetti. Me permettrat-on alors de laisser parler les textes?

\section{Nosotros dos}

La tarde en que me asomé definitivamente a esta ventana una mujer sola con una malla roja tomaba sol entre las sábanas recién tendidas; lo supuse porque había aire y no se movian en la soga. Tenía una toalla de colores vivos atada a la cabeza y en la misma terraza un perro ovejero parecía muerto de un tiro. Me asomé, tuve el mismo miedo de siempre a la altura, el mismo desasosiego ante la posibilidad y tentarme. Ahora busco la manera de acomodar mis libros - les descubro señales de otro tiempo - colgué el mismo Klee del final que se te resistia, y poco a poco la pieza en este quinto piso imprevisible va cobrando un olor que reconozco a fuerza de Particulares Livianos y la yerba dentro del plato que siempre me olvido de sacar.

\section{Nous deux}

Le jour où je me suis penché définitivement à cette fenêtre une femme seule en maillot rouge prenait le soleil entre les draps qu'elle venait d'étendre; je le supposai car il y avait du vent et ils ne bougeaient pas sur la corde. Elle portait nouée sur la tête une serviette aux couleurs 
vives et sur la même terrasse un chien de berger semblait mort d'un coup. Je me penchai, je ressentis comme à l'accoutumée la peur de l'altitude, le même trouble devant la possibilité et me tenter. Maintenant je cherche comment ranger mes livres - j'y découvre les empreintes d'un autre temps - j'ai suspendu ce Klee de la fin qui te résistait, et peu à peu la pièce à ce cinquième étage imprévisible prend une odeur que je reconnais à force de tabac brun et du maté dans l'assiette que j'oublie toujours de vider.

Le traducteur, pas plus que l'auteur de l'original espagnol, n'a de recul pour juger du résultat: en ce sens, Octavio Paz a encore plus raison que tout à l'heure. D'aucuns concluront à une traduction littérale - le respect de la rupture de construction, les pas dans les pas, probablement le même nombre de signes.

Pourtant la phrase française est le fruit d'un long processus de maturation, valsehésitation du traducteur en proie au vertige des mots, cette «tentation» dont parle le texte et à laquelle il se retient pourtant de succomber, car il s'agit bien, avant tout, d'asseoir un texte et de le rendre lisible dans l'étroit sillage de l'imperturbable étranger. Après lecture du français, Néstor m'a adressé ce mot laconique: «Merci pour ton courage!» Un bien grand mot pour un simple exercice de traduction, mais qui montre bien, j'imagine, que la traduction requiert tout à la fois le cœur, les tripes et le bras armé.

Car s'il est simple de s'approprier le langage de l'autre, facile de le faire parler à sa façon, il est autrement plus délicat de respecter la part d'étranger qu'il impose. Le traducteur doit s'attacher à restituer dans son texte l'étrangeté qui constitue le minimum de respect qu'il doit avoir pour le texte à traduire. Or c'est précisément pour être fidèle à cette partie étrange - à moins d'emprunter purement et simplement les mots de l'autre qu'il doit chercher des ressources littéraires, trouver des solutions. Dans Juntacadáveres de Juan Carlos Onetti, la fille Petrus raconte comment elle a gagné aux courses: le Gaucho voulait jouer Sureño, mais elle, dit le texte, habia soñado con el chico de la mujer del Mercado Viejo, tiene tres años, que me seguía hasta casa y yo me iba a dormir y me miraba con la cabeza asomada a la puerta. De veras. Me miraba hasta que lo empujé y cerré la puerta y después lo veía mirando desde la ventana. Entonces, al otro día, miré el diario y dije, Mirón, le voy a jugar a Mirón. Y el Gaucho, cuando le di la plata [y] estuvo riéndose de mí (...) El sabía que ganaba Sureño, sabía que Mirón era un burro.

Le problème posé est la coexistence de deux chevaux de course, dont le second porte un nom qui signifie quelque chose en relation avec le rêve de l'enfant qui regarde la fille depuis la porte. Il faut que le lecteur sente ce lien et comprenne que le rêve de l'enfant curieux amène la fille à préférer au cheval favori un autre cheval dont le nom évoque cette curiosité. Le hic, c'est que les chevaux portent des noms espagnols : peut-on imaginer que le cheval puisse s'appeler «Badaud», «Voyeur» ou «Curieux»? La solution m'a semblé, donc, être de conserver l'étrangeté espagnole, mais alors que Mirón ne peut rien évoquer à l'oreille française, un mot comme Curioso, à cause de la racine latine, évoque bien la curiosité initiale de l'enfant du rêve, et par ailleurs nous savons en France que les chevaux de nos champs de course ont volontiers de ces noms exotiques - n'at-on pas entendu parler du galop de Furioso, de Rabioso, de Solido, voire de De Prisa, de Santa Marina, de Malaspina, etc. ? D'où ma traduction:

(...) j'avais rêvé du garçon de la femme du Vieux Marché, il a trois ans, qui me suivait jusqu'à la maison et j'allais dormir et lui me regardait en glissant la tête par la porte. Vraiment. Il me regardait jusqu'à ce que je le pousse dehors et ferme la porte, et ensuite je le voyais qui regardait par la fenêtre. Alors, le lendemain, j'ai regardé le journal et j'ai dit Curioso, je vais jouer Curioso. Et le Gaucho, quand je lui ai donné l'argent (et qu') il s'est moqué de moi... Il savait que Sureño allait gagner, il savait que Curioso n'était qu'un tocard. 
Est-ce trop s'attarder sur un détail ? Il est parfois plus problématique de traduire un mot qu'un chapitre.

On en jugera peut-être encore mieux à travers un exemple tiré de la pièce de Griselda Gambaro, El despojamiento: une pauvre actrice sur le retour répond à une petite annonce et se trouve, dans une salle d'attente vide, livrée aux brutalités d'un garçon de scène qui la dévêt progressivement; dans le ressassement de sa vie gâchée et de son présent douloureux, elle évoque ou invoque son ami, vaguement souteneur et en tout cas mauvais garçon, qu'elle appelle «Pepe». Imagine-t-on en français pouvoir traduire par «Pépé»? Toute connotation serait perdue et le pathétique basculerait dans le ridicule. Or ce n'est pas simple de transposer un nom: finalement j'ai choisi de l'appeler «Jo». Ainsi : «Espero que Pepe no llegue antes de hora» devient: «J'espère que Jo ne va pas rentrer avant l'heure.» Et juste avant le rideau final: «iAcá espero! (se le petrifica la sonrisa, baja la cabeza, rompe a llorar) ¡Pepe!» devient: «Je suis prête! (Son sourire se pétrifie, elle baisse la tête, éclate en sanglots) Jo!» où, je pense, le monosyllabe doit jaillir de sa poitrine comme un cri, ce que n'aurait sûrement pas permis «Pépé» ou un autre prénom: Roger, Paulot, Riri, que sais-je !

Cette traduction d'un mot, d'un nom propre, nous la retrouvons à travers ce texte magistral - et inédit en espagnol, je crois - de Guillermo Cabrera Infante, Meta-final, que j'ai publié en français sous le titre Métaphynale (revue Le Promeneur, Paris, vol. XLIX, août-septembre 1986). Là le romancier cubain évoque un embaumeur mexicain (qui va se charger de conserver les restes de La Estrella, héroïne de Très tristes tigres, durant son transfert de Veracruz à la Havane) et il écrit «unos amigos del amiguito de La Estrella buscaron al embalsamador adecuado, de nombre Inocente Adecuado, que era el que tenía más fama en México (...). Pero resulta ser que este embalsamador Adecuado era ahora una momia él mismo, un viejo viejo pero muy viejo que apenas se veía a quien embalsamar y tal vez hasta había empezado a autoembalsamarse (...)» Ce que je traduis de la sorte: «des amis du petit ami de La Estrella cherchèrent l'embaumeur adéquat ou plutôt Adequatl (Ignacio Adequatl de son nom), qui était le plus fameux de Mexico (...). Mais il se trouvait que cet embaumeur Adequatl était maintenant une momie de luimême, un vieux vieux mais très vieux qui voyait à peine qui embaumer et peut-être avaitil commencé à s'auto-embaumer».

Le jeu de mot entre le nom Adecuado et l'adjectif «adecuado» qui le précède est facilement rendu en français, mais tout l'arrière-plan mexicain et cette notion d'extrême vieillesse, de momie, pouvait fort bien nous autoriser à remonter plus loin encore, à la civilisation aztèque, et dès lors nous permettre en écho au dieu bien connu Quetzalcoatl, de risquer un curieux Adequatl plus riche de sens et d'allusions. Au lecteur de dire, assurément, s'il se déclare convaincu. Le traducteur ne peut arguer ici que d'une seule justification: la nécessité qu'il s'est imposée de traduire non seulement des mots espagnols, mais aussi et surtout une étrangeté du texte, un caractère insolite que doit ressentir le lecteur, qu'il doit comprendre, car il s'agit toujours, en fin de compte, de l'amener à entrer dans un monde étranger.

Aujourd'hui, au terme de ce trop bref aperçu, que peut-on dire du traducteur? Il n'est plus l'emprunteur, le plagiaire de bonne conscience des temps de la modernité. Il n'est plus ce voleur de culture et le français n'est plus la langue d'un empire où du SaintLaurent à la Terre Adélie tout le monde parlait un français de bon ton. La francophonie nous impose un parler diversifié et souple, une langue pas plus corsetée - ce fut longtemps la réputation de notre langage - qu'une autre. C'est pour cela aussi qu'il est probablement plus facile d'aller vers l'Étranger et de traduire son étrangeté. Certaines traductions de textes latino-américains - ceux où la distorsion par rapport à la languemère est la plus forte - font appel aux ressources des anciennes colonies et des territoires 
d'outre-mer: tel mot antillais, telle parole québécoise, cette expression pied-noir (un des personnages de ¿Quién mató a Palomino Molero?, de Vargas Llosa, s'écrit, à la place du «Chucha de tu madre» réitéré maintes fois, et pour varier les injures, «la mort de tes os», insulte typique importée de l'Algérie française), etc., et le sourcil des sévères correcteurs des maisons d'édition ne se hérisse plus. En ce sens, le traducteur impose d'une certaine façon son propre langage, sa personnalité linguistique. Mais pour être d'autant plus fidèle à son auteur. Cela réclame beaucoup de doigté et une immense sympathie. Mais nous savons bien, au départ comme à l'arrivée, que la traduction est un acte d'amour. 